\title{
SETS OF RICH LINES IN GENERAL POSITION
}

\author{
G. AMIRKHANYAN, A. BUSH, E. CROOT, AND C. PRYBY
}

\begin{abstract}
The Szemerédi-Trotter theorem implies that the number of lines incident to at least $k>1$ of $n$ points in $\mathbb{R}^{2}$ is $O\left(n^{2} / k^{3}+n / k\right)$. J. Solymosi conjectured that if one requires the points to be in a grid formation and the lines to be in general position-no two parallel, no three meeting at a point - then one can get a much tighter bound. We prove a slight variant of his conjecture: for every $\varepsilon>0$ there exists some $\delta>0$ such that for sufficiently large values of $n$, every set of lines in general position, each intersecting an $n \times n$ grid of points in at least $n^{1-\delta}$ places, has size at most $n^{\varepsilon}$. This implies a conjecture of Gy. Elekes about the existence of a uniform statistical version of Freiman's theorem for linear functions with small image sets.
\end{abstract}

\section{INTRODUCTION}

The Szemerédi-Trotter theorem [5] states that, given a set of $n$ points and a set of $m$ lines in $\mathbb{R}^{2}$, the number of incidences (that is, pairs $(p, \ell)$ where $p \in \ell$ ) between these points and lines is $O\left(n^{2 / 3} m^{2 / 3}+m+n\right)$. This bound is sharp up to a constant coefficient. An equivalent form of the theorem states that, given $n$ points and an integer $k>1$, there are $O\left(n^{2} / k^{3}+n / k\right) k$-rich lines (that is, lines that each contain at least $k$ of the $n$ points in the set).

Our goal in this paper is to prove a variant of a conjecture of J. Solymosi [4]:

Theorem 1. For every $\varepsilon>0$, there exists $0<\delta<\varepsilon$ such that for sufficiently large $n=$ $n(\varepsilon, \delta)$, the following holds:

If $A \subseteq \mathbb{R}$ has size $n$, then every set of at least $n^{\varepsilon}$ lines in $\mathbb{R}^{2}$, each of which intersects $A \times A$ in at least $n^{1-\delta}$ points, contains either two parallel lines or three lines with a common intersection point.

The Szemerédi-Trotter theorem gives a bound of $O\left(n^{1+3 \delta}\right) n^{1-\delta}$-rich lines for an arbitrary set of $n^{2}$ points. As a consequence of Solymosi's conjecture, requiring a grid structure in the set of points and general position in the set of lines gives a significant improvement to the Szemerédi-Trotter bound.

We will first prove a weaker form of the conjecture. This form turns out to be sufficient to prove the form above. 
Theorem 2. For every $\varepsilon>0$, there exists $0<\delta<\varepsilon$ such that for sufficiently large $n=$ $n(\varepsilon, \delta)$, the following holds:

If $A \subseteq \mathbb{R},|A|=n$, then every set of at least $n^{1-\varepsilon}$ lines in $\mathbb{R}^{2}$, each of which intersects $A \times A$ in at least $n^{1-\delta}$ points, contains either two parallel lines or $C=C(\varepsilon)>0$ lines with a common intersection point.

We begin by listing the major tools and basic results needed to prove Theorem 1 . In Section 4 we introduce a key result, Theorem 11, describing the behavior of sets of rich lines under self-composition, the main part of which we prove in Section 5. In Section 6, we describe a consequence of Theorem 11 which allows us to extract a set of lines in "nearly" general position from the composition of a set of rich lines with itself. In Section 7, we

prove Theorem 2, and in Section 8, we use the result of Section 6 to showing that Theorem 1 follows from Theorem 22. We conclude in Section 9 by describing an application of Theorem 1: it implies a uniform statistical version of Freiman's theorem, generalized to sets of (affine) linear functions.

\section{MAJOR TOOLS}

In this section we list the theorems we use in this paper. First, we restate the SzemerédiTrotter theorem (in the form we shall use it):

Theorem 3 (Szemerédi-Trotter [6]). If $P$ is any finite set of points and $k \geq 2$, then the number of $k$-rich lines in $P$ is

$$
O\left(\max \left(\frac{|P|^{2}}{k^{3}}, \frac{|P|}{k}\right)\right) .
$$

Before listing the remaining tools we'll be using, let us define some notation. Given two subsets $A, B$ of an additive abelian group, define the sumset by

$$
A+B:=\{a+b: a \in A, b \in B\} .
$$

We further define the iterated sumset by $1 A:=A$ and, for $k>1$,

$$
k A:=(k-1) A+A
$$

Theorem 4 (Plünnecke-Ruzsa [6]). If $A$ is a subset of an additive group such that $|2 A| \leq$ $K|A|$, then $|n A| \leq K^{n}|A|$ for all $n \geq 1$.

If $A, B$ are subsets of an additive abelian group, define the additive energy of $A$ and $B$ as

$$
E(A, B)=\#\left\{\left(a, a^{\prime}, b, b^{\prime}\right) \in A \times A \times B \times B: a+b=a^{\prime}+b^{\prime}\right\} .
$$


Theorem 5 (Balog-Szemerédi-Gowers [6]). Let $A$ be a subset of an additive group. Given $c>0$, there exist constants $C_{1}, C_{2}>0$ dependent only on c such that, if $E(A, A) \geq K^{-c}|A|^{3}$, then there is a subset $A^{\prime} \subseteq A$ such that $\left|A^{\prime}\right| \geq K^{-C_{1}}|A|$ and $\left|2 A^{\prime}\right| \leq K^{C_{2}}\left|A^{\prime}\right|$.

If $A, B$ are subsets of the group of nonzero reals under the operation of multiplication, then we call their sumset (as defined above) the product set, denoted $A . B$, and we call their additive energy (as defined above) multiplicative energy instead.

Theorem 6 (Croot-Hart [3]). For every $c>0$ there exist $\beta>0$ and $k \geq 1$ such that the following holds for all sufficiently large $N$ :

Let $B \subseteq \mathbb{R}$ have size $|B|=N$. If $|B . B| \leq N^{1+\beta}$, then $|k B| \geq N^{c}$.

Let $A$ be a subset of an additive abelian group, and let $A^{k}$ denote the $k$-fold cartesian product of $A$ with itself. If $S \subseteq A^{k}$, then define

$$
\Sigma(S):=\left\{a_{1}+\cdots+a_{k}:\left(a_{1}, \ldots, a_{k}\right) \in S\right\} .
$$

Theorem 7 (Borenstein-Croot [2]). For every $0<\varepsilon<1 / 2$ and $c>1$, there exists $\delta>0$ such that the following holds for all $k, n$ sufficiently large:

Let $A$ be a subset of an additive abelian group with $|A|=n$, and let $S \subseteq A^{k}$. If $|S| \geq|A|^{k-\delta}$ and $|\Sigma(S)|<|A|^{c}$, then there exists a subset $A^{\prime} \subseteq A$ with $\left|A^{\prime}\right| \geq|A|^{1-\varepsilon}$ such that for all $\ell \geq 1,\left|\ell A^{\prime}\right| \leq\left|A^{\prime}\right|^{c(1+\varepsilon \ell)}$.

\section{Preliminary Definitions and Facts}

Let $A \subset \mathbb{R}$ be a finite subset with $|A|=n$. We call a line $\ell$ in $\mathbb{R}^{2} k$-rich if it intersects at least $k$ points in $A \times A$. A line can be at most $n$-rich; we will concern ourselves mainly with lines that are $n^{1-\delta}$-rich for some small positive $\delta$.

If $\ell: y=\lambda x+b$ is a line in $\mathbb{R}^{2}$, then $\ell^{-1}: y=\frac{1}{\lambda} x-\frac{b}{\lambda}$ is the line such that $\ell \circ \ell^{-1}=\ell^{-1} \circ \ell$ is the identity function on $\mathbb{R}^{2}$ (i.e., the line $y=x$ ).

If $L$ is a set of lines in $\mathbb{R}^{2}$, a subset $S \subseteq L$ is a star family if all members of $S$ intersect at some common point. We say $L$ is in general position if no two lines in $L$ are parallel and $L$ has no star families of size 3 . If $L$ has no two parallel lines and no star families of size greater than some constant $C \geq 2$, then we say $L$ is in near-general position.

In the next two lemmas, we establish that many pairs of lines in a set of rich lines can be combined to obtain lines of slightly less richness.

Lemma 8. Given sets $A_{1}, \ldots, A_{k} \subseteq[n]$, each of size at least $n^{1-\delta}$, we must have at least $k^{2} n^{-2 \delta} / 2$ pairs of sets $\left(A_{i}, A_{j}\right)$ with $\left|A_{i} \cap A_{j}\right| \geq n^{1-2 \delta} / 2$. 
Proof. Let $B=\left\{(i, j):\left|A_{i} \cap A_{j}\right| \geq \frac{n^{1-2 \delta}}{2}\right\}$. For a contradiction, suppose $|B|<\frac{1}{2} k^{2} n^{-2 \delta}$. Then

$$
\begin{aligned}
\sum_{i, j}\left|A_{i} \cap A_{j}\right|=\sum_{(i, j) \in B}\left|A_{i} \cap A_{j}\right|+\sum_{(i, j) \in B^{c}}\left|A_{i} \cap A_{j}\right|<n \cdot \frac{1}{2} k^{2} n^{-2 \delta}+ & \\
& \left(k^{2}-\frac{1}{2} k^{2} n^{-2 \delta}\right) \frac{n^{1-2 \delta}}{2}<k^{2} n^{1-2 \delta} .
\end{aligned}
$$

However, letting $d(x):=\#\left\{i \in[k]: x \in A_{i}\right\}$, we have by Cauchy-Schwarz

$$
\sum_{i, j}\left|A_{i} \cap A_{j}\right|=\sum_{x=1}^{n} d(x)^{2} \geq\left(n^{-1 / 2} \sum_{x \in[n]} d(x)\right)^{2}=n^{-1}\left(\sum_{i}\left|A_{i}\right|\right)^{2} \geq k^{2} n^{1-2 \delta} .
$$

Lemma 9. Let $A \subset \mathbb{R}$, and let $L$ be a set of lines in $\mathbb{R}^{2}$ such that each line in $L$ is $n^{1-\delta}$-rich in $A \times A$. Then, for at least $\frac{1}{2}|L|^{2} n^{-2 \delta}$ pairs of lines $\left(\ell, \ell^{\prime}\right) \in L \times L, \ell^{-1} \circ \ell$ is $\frac{1}{2} n^{1-2 \delta}$-rich in $A \times A$.

Proof. For each line $\ell: y=\lambda x+b$, let $X(\ell)=\{x \in A: \lambda x+b \in A\}$, and similarly let $Y(\ell)=\left\{y \in A: \lambda^{-1}(y-b) \in A\right\}$. Observe that $Y(\ell)=X\left(\ell^{-1}\right)$. Thus, for $\left(\ell, \ell^{\prime}\right) \in L \times L$, if $\left|Y(\ell) \cap Y\left(\ell^{\prime}\right)\right| \geq \frac{1}{2} n^{1-2 \delta}$, then $\ell^{-1} \circ \ell^{\prime}$ is $\frac{1}{2} n^{1-2 \delta}$-rich in $A \times A$. Observe that each $Y(\ell)$ has size at least $n^{1-\delta}$. By Lemma 8 , at least $\frac{1}{2}|L|^{2} n^{-2 \delta}$ pairs of sets $\left(Y(\ell), Y\left(\ell^{\prime}\right)\right)$ have intersection of size at least $\frac{1}{2} n^{1-2 \delta}$.

We define the operation $*$ by $\ell_{1} * \ell_{2}=\ell_{1}^{-1} \circ \ell_{2}$. This formalizes the notion described earlier of combining rich lines in $L$ to form new rich lines (at the cost of a small amount of richness). Given two sets $L, L^{\prime}$ of $n^{1-\delta}$-rich lines, we would like to consider the set of lines $\ell * \ell^{\prime}$ which retain a large amount of richness in $A \times A$.

$$
\left\{\ell * \ell^{\prime}: \ell \in L, \ell^{\prime} \in L^{\prime},\left|\ell * \ell^{\prime} \cap(A \times A)\right| \geq n^{1-2 \delta} / 2\right\} .
$$

Corollary 10. Given a set $L$ of lines which are $n^{1-\delta}$-rich in $A \times A$, there exist at least $\frac{1}{2}|L| n^{-2 \delta}$ distinct lines of the form $\ell * \ell^{\prime}$ which are $\frac{1}{2} n^{1-2 \delta}$-rich in $A \times A$.

Proof. There can be at most $|L|$ pairs which map to a given line in $L * L$, or else there exists $\ell_{1} \in L$ and $\ell_{2} \neq \ell_{3}$ such that $\ell_{1}^{-1} \circ \ell_{2}=\ell_{1}^{-1} \circ \ell_{3}$, a contradiction. By Lemma 9, the result therefore follows.

In addition to the richness of our new lines in $A \times A$, we will want to have control over the number of pairs $\left(\ell_{1}, \ell_{2}\right)$ which map to the same line under $*$. Let $\mathcal{P}(\ell)$ denote the set of pairs $\left(\ell_{1}, \ell_{2}\right)$ such that $\ell_{1} * \ell_{2}=\ell$; if $X$ is a set of lines, let $\mathcal{P}(X)$ be the union of the sets 
$\mathcal{P}(\ell)$ over all $\ell \in X$. For each $0 \leq i \leq\left\lceil\log _{2}|L|\right\rceil$, let $L_{i}$ be the set of those lines $\ell$ in the set (1) such that

$$
2^{i-1}<|\mathcal{P}(\ell)| \leq 2^{i}
$$

and let

$$
N_{i}=\sum_{\ell \in L_{i}}|\mathcal{P}(\ell)|
$$

Then

$$
N_{0}+N_{1}+\cdots+N_{K}=\#\left\{\left(\ell, \ell^{\prime}\right):\left|\ell * \ell^{\prime} \cap(A \times A)\right| \geq \frac{1}{2} n^{1-2 \delta}\right\} \geq \frac{1}{2}|L|^{2} n^{-2 \delta}
$$

by Lemma 9 . By the pigeonhole principle, at least one $N_{i}$ satisfies

$$
N_{i} \geq \frac{|L|^{2} n^{-2 \delta}}{2 \log _{2}|L|} .
$$

For the maximal such $i$, we define $L * L$ to be

$$
L * L:=\left\{\ell * \ell^{\prime}:\left(\ell, \ell^{\prime}\right) \in \mathcal{P}\left(L_{i}\right)\right\} .
$$

If $L$ is a set of $n^{1-\delta}$ rich lines, we recursively define the sequence of $j$-fold $*$ operations on $L$ as follows: take $L^{* 2}:=L * L$ and $L^{* j}:=L^{*(j-1)} * L^{*(j-1)}$. We remark that the operation $*$ is not associative: for example, $(L * L) *(L * L)$ will not in general equal $((L * L) * L) * L$.

\section{Lines in Near-General Position}

The following theorem illustrates the behavior of a near-general position set of lines under the operation of $*$.

Theorem 11. For all $0<\varepsilon<1$, there exists $0<\alpha_{0}<\varepsilon$ such that for all $0<\alpha<\alpha_{0}$, there exists $0<\delta_{0}<\alpha$ such that for all $0<\delta<\delta_{0}$ and for finite sets $A$ with $|A|=n$ sufficiently large, the following holds:

If $L$ is a set of at least $n^{\varepsilon}$ lines in near-general position (with star families bounded by some constant $C=C(\varepsilon, \alpha)>0$ ) which are $n^{1-\delta}$-rich in $A \times A$, then:

(i) If $L * L$ contains a family $P$ of parallel lines, then $|P| \leq 2|L * L| n^{2 \delta} /|L|$.

(ii) If $L * L$ contains a star family $S$, then $|S| \leq 2 C|L * L| n^{2 \delta} /|L|$.

(iii) If $P_{\lambda}$ denotes the set of lines in $L * L$ with common slope $\lambda$, then $\left|P_{\lambda}\right| \geq n^{\alpha}$ for at most $n^{\alpha}$ numbers $\lambda$.

(iv) If $S_{p}$ denotes the set of lines in $L * L$ with common meeting point $p$, then $\left|S_{p}\right| \geq n^{\alpha}$ for at most $n^{\alpha}$ points $p$.

Conditions (i), (ii), and (iv) will be shown in this paper. Condition (iii) is shown to hold in [1]. 
The proofs of conditions (i) and (ii) are similar. Given a line $\ell \in L * L$, recall that $\mathcal{P}(\ell)$ denotes the set of pairs $\left(\ell_{1}, \ell_{2}\right) \in L \times L$ such that $\ell_{1} * \ell_{2}=\ell$. If $X \subset L * L$, define $\mathcal{P}(X):=\bigcup_{\ell \in X} \mathcal{P}(\ell)$.

\subsection{Large Families of Parallel Lines.}

Proof of Theorem 11 (i). Suppose there is a family $P \subseteq L * L$ of parallel lines with

$$
|P|>\frac{2|L * L|}{|L|} n^{2 \delta} \geq \frac{|L|}{2^{i}}
$$

where $i$ is the maximal index between 0 and $\left\lceil\log _{2}|L|\right\rceil$ such that

$$
\#\left\{\ell \in L * L: 2^{i-1}<|\mathcal{P}(\ell)| \leq 2^{i}\right\} \geq \frac{|L|^{2} n^{-2 \delta}}{2 \log _{2}|L|} .
$$

Then the total number of pairs mapping to lines in $P$ under $*$ will be

$$
|\mathcal{P}(P)|=\sum_{\ell \in P}|\mathcal{P}(\ell)|>|P| 2^{i}>|L| .
$$

Since the lines of $L$ have distinct slopes, it follows that there exist two distinct pairs $(\lambda x+$ $\left.b, \lambda^{\prime} x+b^{\prime}\right)$ and $\left(\lambda x+b, \lambda^{\prime \prime} x+b^{\prime \prime \prime}\right)$ which each map to some line in $P$. But then $\lambda^{\prime}=\lambda^{\prime \prime}$, and by the distinctness of the pairs it follows that $b^{\prime} \neq b^{\prime \prime \prime}$. Thus, two lines in $L$ are parallel, contradicting the hypothesis that they are in near-general position.

\subsection{Large Star Families.}

Proof of Theorem 11(ii). It is sufficient to consider the case that the lines in $S$ intersect on the $y$-axis. If not, suppose the center of $S$ is $\left(x_{0}, y_{0}\right)$, and consider the grid $A^{\prime} \times A^{\prime}$, where $A^{\prime}$ is the translate $A-x_{0}$. Suppose the pair $\left(\ell_{1}, \ell_{2}\right)$ of $n^{1-\delta}$-rich lines in $A \times A$ maps to a line in $S$, where $\ell_{1}: y=\lambda_{1} x+b_{1}$ and $\ell_{2}: y=\lambda_{2} x+b_{2}$. Then

$$
\ell_{1} * \ell_{2}: y=\frac{\lambda_{2}}{\lambda_{1}} x+\frac{b_{2}-b_{1}}{\lambda_{1}}
$$

contains the point $\left(x_{0}, y_{0}\right)$. Let $\ell_{1}^{\prime}, \ell_{2}^{\prime}$ be the translates of $\ell_{1}, \ell_{2}$ down by $x_{0}$ and left by $x_{0}$; then

$$
\ell_{1}^{\prime}: y=\lambda_{1} x+\lambda_{1} x_{0}+b_{1}-x_{0} \quad \text { and } \quad \ell_{2}^{\prime}: y=\lambda_{2} x+\lambda_{2} x_{0}+b_{2}-x_{0} .
$$

So $\left(\ell_{1}^{\prime}, \ell_{2}^{\prime}\right)$ maps to

At $x=0$, we have

$$
\ell_{1}^{\prime} * \ell_{2}^{\prime}: y=\frac{\lambda_{2}}{\lambda_{1}} x+\frac{\left(\lambda_{2}-\lambda_{1}\right) x_{0}+b_{2}-b_{1}}{\lambda_{1}} .
$$

$$
y=\frac{\lambda_{2}}{\lambda_{1}} x_{0}+\frac{b_{2}-b_{1}}{\lambda_{1}}-x_{0}=y_{0}-x_{0} .
$$


That is to say, $\left(\ell_{1}^{\prime}, \ell_{2}^{\prime}\right)$ maps to a rich line passing through the point $\left(0, y_{0}-x_{0}\right)$. Thus, given a star family of lines $\frac{1}{2} n^{1-2 \delta}$-rich in $A \times A$, we can construct a new $\frac{1}{2} n^{1-2 \delta}$-rich star family of the same size in the translated grid $A^{\prime} \times A^{\prime}$ whose center lies on the $y$-axis.

Now suppose there is a star family $S \subseteq L * L$ centered at $\left(0, y_{0}\right)$ with

$$
|S|>2 C \frac{|L * L|}{|L|} n^{2 \delta} \geq C \frac{|L|}{2^{i}}
$$

(where $i$ is taken as in the previous proof). Then the total number of preimages for lines in $S$ will be

$$
|\mathcal{P}(S)|=\sum_{\ell \in S}|\mathcal{P}(\ell)|>|S| 2^{i}>C|L| .
$$

Since the lines of $L$ have distinct slopes, it follows that there exist $C+1$ distinct pairs in $L \times L$ mapping to $S$ such that the lines in the first coordinate of the pairs are the same:

$$
\left(\lambda x+b, \lambda_{1} x+b_{1}\right),\left(\lambda x+b, \lambda_{1} x+b_{2}\right), \ldots,\left(\lambda x+b, \lambda_{C+1} x+b_{C+1}\right) .
$$

Since the $y$-intercepts of the output lines are the same, it follows that

$$
\frac{b_{1}-b}{\lambda}=\frac{b_{2}-b}{\lambda}=\cdots=\frac{b_{C+1}-b}{\lambda}=y_{0} ;
$$

in other words, $b_{1}=b_{2}=\cdots=b_{C+1}=\lambda y_{0}+b$. Thus, $L$ contains $C+1$ lines with a common $y$-intercept, contradicting the hypothesis that $L$ is in near-general position with star families bounded in size by $C$.

\section{Star Families of Moderate Size}

Moving towards a proof of case (iv) of Theorem 11, we begin with a technical combinatorial result.

Lemma 12. For every $c>0$ there exist $\alpha>0$ and $k \geq 1$ such that the following holds for all sufficiently large $M$ : suppose that $A_{1}, A_{2}, \ldots, A_{k} \subseteq \mathbb{R}$ with $\left|A_{i}\right|=M$ and $\left|A_{i} . A_{i}\right| \leq M^{1+\alpha}$ for all $i=1, \ldots, k$. If $S \subseteq A_{1} \times A_{2} \times \cdots \times A_{k}$ has size $|S| \geq M^{k-\alpha}$, then $|\Sigma(S)| \geq M^{c}$.

Proof. Let $A_{1}, \ldots, A_{k}, S$ be sets as in the statement of the lemma. Suppose that $|\Sigma(S)|<M^{c}$, where we assume that $M$ is large in terms of $k, c$, and $\alpha$ - say, $M>2^{2^{c+k+\alpha^{-1}+100}}$.

Apply Theorem [7, taking $\alpha<\delta / 2, \varepsilon>0$ sufficiently small (to be determined later), and $A=A_{1} \cup \cdots \cup A_{k}$. Take $N=|A|$, observing that $M \leq N \leq k M$. Note that $S \subset A^{k}$,

$$
|S| \geq M^{k-\alpha}>(N / k)^{k-\alpha}>N^{k-\delta}, \quad \text { and } \quad|\Sigma(S)|<M^{c} \leq N^{c} .
$$

Then there is a subset $A^{\prime} \subseteq A$ with $\left|A^{\prime}\right| \geq N^{1-\varepsilon}$ such that for all $\ell \geq 1,\left|\ell A^{\prime}\right|<N^{c(1+\varepsilon \ell)}$. 
By the pigeonhole principle, $A^{\prime}$ intersects some $A_{j}, 1 \leq j \leq k$, in a set of size at least $\left|A^{\prime}\right| / k>N^{1-\varepsilon} / k$. Let $A^{\prime \prime}$ be that intersection, and note that this set satisfies the following:

$$
\left|A^{\prime \prime}\right|>N^{1-\varepsilon} / k, \quad\left|A^{\prime \prime} . A^{\prime \prime}\right| \leq\left|A_{j} \cdot A_{j}\right| \leq M^{1+\alpha}, \quad \text { and } \quad\left|\ell A^{\prime \prime}\right|<N^{c(1+\varepsilon \ell)} .
$$

Expressing all of this in terms of $N^{\prime}=\left|A^{\prime \prime}\right|$, we get

$$
\left|A^{\prime \prime} . A^{\prime \prime}\right| \leq M^{1+\alpha} \leq\left(k N^{\prime}\right)^{(1+\alpha) /(1-\varepsilon)} \quad \text { and } \quad\left|\ell A^{\prime \prime}\right|<\left(k N^{\prime}\right)^{c(1+\varepsilon \ell) /(1-\varepsilon)} .
$$

For $k \geq 1$, if $\ell$ is sufficiently large in terms of $c$, and if $\alpha$ and $\varepsilon$ are sufficiently small in terms of $\ell$ and $k$, then these inequalities contradict Theorem [6.

From this lemma we prove a corollary which will have a direct application to the proof of condition (iv) of Theorem 11.

Lemma 13. There is an absolute constant $c>0$ such that for every $\varepsilon>0$ there exists $0<\alpha_{0}<\varepsilon$ such that for all $0<\alpha<\alpha_{0}$, there exists $0<\delta_{0}<\alpha$ such that the following holds for all $0<\delta<\delta_{0}$ and sufficiently large $n=n(\varepsilon, \alpha, \delta)$ :

If $\left\{C_{1}, \ldots, C_{k}\right\}$ is a collection of sets of real numbers such that for all $i,\left|C_{i}\right| \geq n^{\alpha}$ and $\left|C_{i} . C_{i}\right| \leq\left|C_{i}\right|^{1+c_{1} \delta}, B$ is a set of real numbers with $|B| \geq n^{\varepsilon}$, and $x_{1}, \ldots, x_{k} \in \mathbb{R}$ are distinct constants such that for all $i$ and for each $\lambda \in C_{i}$, there are at least $|B|^{1-c \delta}$ pairs $\left(b, b^{\prime}\right) \in B \times B$ satisfying $\lambda\left(b-x_{i}\right)=b^{\prime}-x_{i}$, then $k<n^{\alpha-c \delta}$.

First we need another lemma:

Lemma 14. Suppose that $d_{i, j}, i=1, \ldots, k$ and $j=1, \ldots, N$ are real numbers satisfying $0 \leq d_{i, j} \leq$ L. If $C \geq 0$ is defined by

$$
\sum_{i=1}^{k} \sum_{j=1}^{N} d_{i, j}=C L k N
$$

then there exists $i \in[k]$ such that for at least $k C^{2} /\left(2-C^{2}\right)$ indices $i^{\prime} \in[k]$, we have

$$
\sum_{j=1}^{N} d_{i, j} d_{i^{\prime}, j}>\frac{1}{2} C^{2} L^{2} N
$$

Proof of Lemma. By the Cauchy-Schwarz inequality,

$$
\sum_{1 \leq i, i^{\prime} \leq k} \sum_{j=1}^{N} d_{i, j} d_{i^{\prime}, j}=\sum_{j=1}^{N}\left(\sum_{i=1}^{k} d_{i, j}\right)^{2} \geq \frac{1}{N}\left(\sum_{j=1}^{N} \sum_{i=1}^{k} d_{i, j}\right)^{2}=C^{2} L^{2} k^{2} N .
$$

In particular, there must exist some $i \in[k]$ such that

$$
\sum_{i^{\prime}=1}^{k} \sum_{j=1}^{N} d_{i, j} d_{i^{\prime}, j} \geq C^{2} L^{2} k N
$$


Fixing such an $i$, let $T$ denote the number of indices $i^{\prime} \in[k]$ for which

$$
\sum_{j=1}^{N} d_{i, j} d_{i^{\prime}, j} \leq \frac{1}{2} C^{2} L^{2} N .
$$

Then

SO

$$
\frac{1}{2} T C^{2} L^{2} N+(k-T) L^{2} N \geq C^{2} L^{2} k N
$$

$$
T \leq k \frac{1-C^{2}}{1-C^{2} / 2}
$$

Thus, for at least

$$
k-T \geq \frac{k C^{2}}{2-C^{2}}
$$

indices $i^{\prime} \in[k]$, (2) holds.

Proof of Lemma 13. By a dyadic pigeonhole argument, there exists a subcollection of the set $\left\{C_{1}, \ldots, C_{k^{\prime}}\right\}$ with size $\frac{k^{\prime}}{\log _{2}(n)}$ and an integer $L \geq n^{\alpha}$ such that $L \leq\left|C_{i}\right| \leq 2 L$ for each $C_{i}$ in the subcollection. Let $k$ be the number of elements in this subcollection, and reindex so that $C_{1}, \ldots, C_{k}$ are the sets making up the subcollection.

For each $i=1, \ldots, k$, construct the directed bipartite graph $G_{i}$ on vertex set $B_{1} \sqcup B_{2}$ where $B_{1}=B_{2}=B$ and where $\left(b, b^{\prime}\right)$ is an edge if there exists $\lambda \in C_{i}$ such that $\lambda\left(b-x_{i}\right)=b^{\prime}-x_{i}$. Letting $N=|B|$, the sum of the out-degrees in $B_{1}$ (and the sum of the in-degrees in $B_{2}$ ) is at least $L N^{1-O(\delta)}$ by our hypotheses on the size of $C_{i}$.

If $G$ is a directed graph, define $\widetilde{G}$ to be the graph obtained by reversing the orientation of each of $G$ 's edges.

If $G$ and $G^{\prime}$ are two $\left(2^{t}+1\right)$-partite directed graphs whose vertex sets are $B_{1} \sqcup \cdots \sqcup B_{2^{t}+1}$, where $B_{1}=\cdots=B_{2^{t}+1}=B$, then define the $\left(2^{t+1}+1\right)$-partite directed graph $G \wedge G^{\prime}$ as follows: Let $V=B_{1} \sqcup \cdots \sqcup B_{2^{t+1}+1}$. For $m=1, \ldots, 2^{t}$, let $\left(b_{j}, b_{j^{\prime}}\right) \in B_{m} \times B_{m+1}$ be an edge in $G \wedge G^{\prime}$ if and only if $\left(b_{j}, b_{j^{\prime}}\right) \in B_{m} \times B_{m+1}$ is an edge in $G$. For $m=2^{t}+1, \ldots, 2^{t+1}$, let $\left(b_{j}, b_{j^{\prime}}\right) \in B_{m} \times B_{m+1}$ be an edge in $G \wedge G^{\prime}$ if and only if $\left(b_{j}, b_{j^{\prime}}\right) \in B_{m-2^{t}} \times B_{m+1-2^{t}}$ is an edge in $G^{\prime}$.

Define $G_{i_{1}, i_{2}}$ to be $G_{i_{1}} \wedge \widetilde{G}_{i_{2}}$. By Lemma 14 (taking $C=N^{-O(\delta)}$ ) there is an index $1 \leq i_{1} \leq k$ such that for at least $k N^{-O(\delta)}$ indices $1 \leq i_{2} \leq k$,

$$
\sum_{j=1}^{N} d_{i_{1}, j} d_{i_{2}, j} \geq L^{2} N^{1-O(\delta)}
$$

where $d_{i, j}$ is the number of directed edges in $G_{i}$ terminating at $b_{j} \in B_{2}$. This sum then counts the total number of paths of length 2 in $G_{i_{1}, i_{2}}$, so the average number of paths in $G_{i_{1}, i_{2}}$ terminating at a particular $b \in B$ is at least $L^{2} N^{-O(\delta)}$. 
Now, fixing $i_{1}, \ldots, i_{t-1}$, we can apply Lemma 14 again to form a $\left(2^{t}+1\right)$-partite graph

$$
G_{i_{1}, \ldots, i_{t+1}}=G_{i_{1}, \ldots, i_{t-1}, i_{t}} \wedge \widetilde{G}_{i_{1}, \ldots, i_{t-1}, i_{t+1}}
$$

with $L^{2^{t}} N^{1-O(\delta)}$ length-2t $2^{t}$ paths corresponding to $2^{t}$-tuples $\left(\lambda_{1}, \ldots, \lambda_{2^{t}}\right)$ such that $\lambda_{4 m+1} \in C_{i_{1}}$ and $\lambda_{4 m} \in C_{i_{1}}^{-1}$ for all $m$.

Using the fact that

$$
\lambda_{j}\left(\beta_{j}-x_{j}\right)=\beta_{j+1}-x_{j},
$$

or, equivalently,

$$
\lambda_{j} \beta_{j}=\beta_{j+1}-x_{j}+\lambda_{j} x_{j},
$$

each of these $2^{t}$-tuples corresponds to an expression of the form:

$$
\begin{aligned}
\lambda_{2^{t}} \cdots \lambda_{1} \beta_{1} & =\lambda_{2^{t}} \cdots \lambda_{2}\left(\beta_{2}-x_{1}+\lambda_{1} x_{1}\right) \\
& =\lambda_{2^{t}} \cdots \lambda_{2} \beta_{2}-\lambda_{2^{t}} \cdots \lambda_{2} x_{1}+\lambda_{2^{t}} \cdots \lambda_{1} x_{1} \\
& \vdots \\
& =\left(\beta_{2^{t}+1}-x_{2^{t}}\right)+\sum_{y=1}^{2^{t}}\left[\prod_{j=y}^{2^{t}} \lambda_{j}\right]\left(x_{y}-x_{y-1}\right),
\end{aligned}
$$

where we define $x_{0}=0$.

By the pigeonhole principle, there exists a choice of $\beta_{1}$ and of the variables $\lambda_{j}, j \not \equiv$ $1(\bmod 4)$, for which there are at least $L^{2^{t-2}} N^{-O(\delta)}$ paths in the $\left(2^{t}+1\right)$-partite graph starting at $\beta_{1}$ and utilizing the edges specified by the selected $\lambda_{j}$ (leaving at least $L^{2^{t-2}} N^{-O(\delta)}$ free choices of edges). Fixing such a $\beta_{1}$ and the variables $\lambda_{j}$ except $\lambda_{4 s-3} \in C_{i}$ for all $1 \leq s \leq 2^{t-2}$, the left-hand side of the equality

$$
\lambda_{2^{t}} \cdots \lambda_{1} \beta_{1}=\left(\beta_{2^{t}+1}-x_{2^{t}}\right)+\sum_{y=1}^{2^{t}}\left[\prod_{j=y}^{2^{t}} \lambda_{j}\right]\left(x_{y}-x_{y-1}\right),
$$

is an expression contained in the set

$$
C_{i}^{2^{t-2}} \cdot \beta_{1} \cdot \prod_{\substack{1 \leq j \leq 2^{t} \\ j \neq 1(\bmod 4)}} \lambda_{j}
$$

which by Theorem 4 has size at most $\left|C_{i}\right|^{1+O_{t}(\delta)}$. Now we rewrite the right-hand-side, by grouping the terms indexed by $y$ into groups of four, starting at $y=4 r+2$ : a typical such 
group will have sum

$$
\begin{aligned}
\lambda_{4 r+5} \lambda_{4 r+6} \cdots \lambda_{2^{t}}\left(\lambda_{4 r+2} \lambda_{4 r+3} \lambda_{4 r+4}\left(x_{4 r+2}-x_{4 r+1}\right)\right. & \\
& +\lambda_{4 r+3} \lambda_{4 r+4}\left(x_{4 r+3}-x_{4 r+2}\right) \\
& \left.+\lambda_{4 r+4}\left(x_{4 r+4}-x_{4 r+3}\right)+x_{4 r+5}-x_{4 r+4}\right) .
\end{aligned}
$$

Conveniently, all the terms in the parentheses involve products of $\lambda_{j} \mathrm{~s}$, where $j \not \equiv 1(\bmod 4)$. We can assume here that all of $x_{4 r+1}, x_{4 r+2}, x_{4 r+3}, x_{4 r+4}$ are distinct (the number of expressions resulting in duplications is small compared to the total number); and so, we have that $x_{4 r+2}-x_{4 r+1}, x_{4 r+3}-x_{4 r+2}, x_{4 r+4}-x_{4 r+3}$, and $x_{4 r+5}-x_{4 r+4}$ are all non-zero. It follows, therefore, that for any choice of two of the parameters among $\lambda_{4 r+2}, \lambda_{4 r+3}, \lambda_{4 r+4}$, there can be at most one possible choice of the remaining parameter that can make the expression (44) equal to 0 . In fact, the number of paths through the graph resulting in a selection of the $\lambda_{j}$ 's where at least one of the $2^{t-2}$ four-tuples equals 0 is at most $L^{2^{t}-1} N^{1-O(\delta)}$. But since there are many more paths than this, we can assume that there is a choice for the $\lambda_{j}$, $j \not \equiv 1(\bmod 4)$ where all the four-tuples are non-zero. Re-expressing the right-hand-side of (3) in terms of these four-tuples, for this fixed choice of $\lambda_{j}, j \neq \equiv 1(\bmod 4)$, we find that it is contained in the set

$$
\beta_{2^{t}+1}-x_{2^{t}}+\sum_{j=1}^{2^{t-2}} \kappa_{j} C_{i}^{4 j}
$$

where $\kappa_{j} \neq 0$ are constants. Furthermore, it turns out that for at least $\left|C_{i}\right|^{2^{t-2}-O(\delta)}$ vectors $\left(c_{1}, c_{2}, \ldots, c_{2^{t-2}}\right) \in C_{i} \times C_{i}^{2} \times \cdots \times C_{i}^{2^{t-2}}$, this expression is among the expressions in the righthand-side of (3) that we can produce by Ruzsa-Plunnecke (since $\mid C_{i}$. $C_{i}|\approx| C_{i} \mid$ ). Applying Lemma 12, then, we quickly find that for $t$ large enough, the number of right-hand-side expressions exceeds $L^{2}$. This contradicts the fact that the numberof left-hand side expressions is bounded by $L^{1+O_{t}(\delta)}$. This contradiction finishes the proof.

Now, we may finally establish that if $L$ is in near-general position, then $L * L$ does not contain too many star families of "moderate" size.

Proof of Theorem 11(iv). Suppose that there exist $k=n^{\alpha}$ star families $S_{1}, \ldots, S_{k} \subseteq L * L$ with $\left|S_{i}\right| \geq n^{\alpha}$ for all $i$. Under this assumption, we will construct sets $B, C_{1}, \ldots, C_{k}$ and distinct constants $x_{1}, \ldots, x_{k} \in \mathbb{R}$ such that $\left|C_{i}\right| \geq n^{\alpha}$, such that $\left|C_{i} \cdot C_{i}\right| \leq\left|C_{i}\right|^{1+O\left(\delta^{\prime}\right)}$ (for $\left.\delta^{\prime} \ll \alpha\right)$ and such that for all $i$ and for all $\lambda \in C_{i}$, we have $|B|^{1-O\left(\delta^{\prime}\right)}$ pairs $\left(b, b^{\prime}\right) \in B \times B$ such that $\lambda\left(b-x_{i}\right)=b^{\prime}-x_{i}$. This construction is forbidden by Lemma 13, giving us a contradiction.

Begin by taking the $x_{i}$ to be the $x$-coordinates of the centers of the star families $S_{1}, \ldots, S_{k}$ in $L * L$. Our first difficulty will be to show that the $x_{i}$ are distinct. Indeed, this may not be the 
case, for it is possible that many of the star families lie on common vertical lines. However, suppose that there are $K$ distinct vertical lines on which there are star families. Then there is some such line with at least $n^{\alpha} / K$ star families on it. Now, since a line $\ell: y=\lambda x+b$ is in $L * L$ if and only if its inverse $\ell^{-1}: x=\lambda y+b$ is also in $L * L$, it follows that there is a horizontal line with $n^{\alpha} / K$ star families on it, implying there are at least that many distinct vertical lines. Hence, $K \geq n^{\alpha} / K$, so $K \geq n^{\alpha / 2}$. By choosing one star family from each vertical line and ignoring the rest (and reducing $\alpha$ to $\alpha / 2$ ) we attain distinct $x$-coordinates for the centers of the star families.

Now, fix a star family $S_{i}$, and let $\Lambda_{i}$ be the set of slopes of the lines in $S_{i}$. Observe that lines in $S_{i}^{* 2}:=S_{i} * S_{i}$ will have slopes in the ratio set $Q_{i}:=\Lambda_{i} / \Lambda_{i}$, lines in $S_{i}^{* 3}:=S_{i}^{* 2} * S_{i}^{* 2}$ will have slopes in $Q_{i}^{2}=\left(\Lambda_{i} / \Lambda_{i}\right)^{2}$, and (in general) lines in $S_{i}^{*(j+2)}, j \geq 0$, will have slopes in the set $Q_{i}^{2^{j}}$. Now, not all elements of $Q_{i}^{2^{j}}$ will be slopes of lines in $S_{i}^{*(j+2)}$ (because some combined lines will not be rich enough in $A \times A)$. Let $M_{i, j} \subset Q_{i}^{2^{j}}$ be the set of slopes of lines in $S_{i}^{*(j+2)}$.

Observe that $S_{i} * S_{i}$ is itself a star family centered at $\left(x_{i}, x_{i}\right)$, and $S_{i} * S_{i}$ contains the line $y=x$. Therefore, $S_{i}^{* j} \subseteq S_{i}^{*(j+1)}$ for all $j \geq 2$; hence $M_{i, j-1} \subseteq M_{i, j}$ for all $j \geq 1$. Moreover, since $y=x$ is in $S_{i} * S_{i}, M_{i, j}$ is closed under taking reciprocals for all $j$. Further note that lines in $S_{i}^{* j}$ will be $n^{1-2^{O(j)}} \delta$-rich in $A \times A$.

Let $\delta^{\prime}>0$ be a parameter such that $0<\delta<\delta^{\prime}<\alpha$. Suppose $\left|M_{i, j+1}\right| \geq\left|M_{i, j}\right| n^{\delta^{\prime} \alpha}$ for all $j$ up to $m=\left\lfloor 2 / \delta^{\prime} \alpha\right\rfloor$. Redefining $\delta$ if necessary, we may take $1-5^{m} \delta>0$. For sufficiently large $n$, we then have

$$
\left|M_{i, m+1}\right| \geq n^{\alpha+m \delta^{\prime} \alpha} \geq n^{2} .
$$

But, since each element of $M_{i, m+1}$ corresponds to a distinct rich line in $A \times A$, this violates Theorem 3. Therefore there exists some $j=j(i)<2 / \delta^{\prime} \alpha$ such that

$$
\left|M_{i, j+1}\right|<\left|M_{i, j}\right| n^{\delta^{\prime} \alpha} \leq\left|M_{i, j}\right|^{1+\delta^{\prime}} .
$$

Therefore, by Lemma 9, there are at least $\left|M_{i, j}\right|^{2-O\left(\delta^{\prime}\right)}$ pairs $\left(m, m^{\prime}\right) \in M_{i, j} \times M_{i, j}$ such that $m / m^{\prime} \in M_{i, j+1}$. So the multiplicative energy of $M_{i, j}$ satisfies

$$
E\left(M_{i, j}, M_{i, j}\right) \geq\left|M_{i, j}\right|^{3-O\left(\delta^{\prime}\right)} .
$$

By Theorem 5, we conclude there is a subset $M_{i}^{\prime} \subseteq M_{i, j}$ with small multiplicative doubling: $\left|M_{i}^{\prime} . M_{i}^{\prime}\right| \leq\left|M_{i}^{\prime}\right|^{1+O\left(\delta^{\prime}\right)}$. Let $S_{i}^{\prime}$ be those lines $\ell \in S_{i}^{*(j+2)}$ such that the slope of $\ell$ is in $M_{i}^{\prime}$.

Now, let $\ell: \lambda x+\left(x_{i}-\lambda x_{i}\right)=\lambda\left(x-x_{i}\right)+x_{i}$ be a line in the stable star family $S_{i}^{\prime}$. Since this line is rich in $A \times A$, there are $|A| n^{-O\left(\delta^{\prime}\right)}$ pairs $\left(a, a^{\prime}\right) \in A \times A$ such that $\lambda\left(a-x_{i}\right)=a^{\prime}-x_{i}$. Taking $C_{i}=M_{i}^{\prime}$ and $B=A$, this is the forbidden construction we desired above. 


\section{Constructing a Near-General Position Set of Lines}

Using Theorem 11, we can extract a subset of lines in $L * L$ which is in near-general position: the subset will contain no two parallel lines, and all star families in the subset have size bounded by a constant $C=C(\varepsilon, \alpha)$ independent of $n$.

Corollary 15. For all $0<\varepsilon<1$ there exists $0<\alpha_{0}<\varepsilon$ such that, for all $0<\alpha<\alpha_{0}$, there exists $0<\delta_{0}<\alpha$ such that for all $0<\delta<\delta_{0}$ and for sufficiently large $n$, the following holds:

Let $A \subseteq \mathbb{R}$ be a finite set with $|A|=n$, and let $L$ be a set of at least $n^{\varepsilon}$ lines which are all $n^{1-\delta}$-rich in $A \times A$. If $L$ contains no parallel lines and all star families in $L$ are bounded above in size by $C=C(\varepsilon, \alpha)$, then there exists a subset $R \subseteq L * L$ such that

- $|R| \geq|L| n^{-c \alpha}$ for some absolute constant $c$,

- $R$ contains no two lines which are parallel, and

- at most $k=\lceil\varepsilon / \alpha\rceil$ lines of $R$ pass through any given point of $\mathbb{R}^{2}$.

We need a short lemma, which is easily proved by induction.

Lemma 16. Let $k$ be a nonnegative integer and $0<\gamma<1$. Then

$$
\lim _{x \rightarrow \infty} x^{k \gamma} \cdot \frac{\left(\begin{array}{c}
x \\
x^{1-\gamma}-k
\end{array}\right)}{\left(\begin{array}{c}
x \\
x^{1-\gamma}
\end{array}\right)}=1 .
$$

Proof of Corollary 15. By Theorem 11(iii), there are at most $n^{\alpha}$ families of parallel lines in $L * L$ with size greater than $n^{\alpha}$. By Theorem 11(i), none of these families can have size greater than $2|L * L| n^{2 \delta} /|L|$. Thus, deleting all of these lines from $L * L$ leaves us with a set of at least

$$
|L * L|-\frac{2|L * L| n^{\alpha+2 \delta}}{|L|}>\frac{1}{2}|L * L|
$$

lines.

The remaining families of parallel lines in this set have size at most $n^{\alpha}$, and these families are all disjoint. By picking a single representative from each family, we form a subset of $L * L$ of at least $\frac{1}{2}|L * L| n^{-\alpha}$ lines, no two of which are parallel. Invoking Theorem 11(ii) and (iv), we remove from this subset all star families of size greater than $n^{\alpha}$ to leave us with a subset $L^{\prime}$ with at least $\frac{1}{4}|L * L| n^{-\alpha}$ lines.

By Corollary 10, there are at least $|L| n^{-3 \delta}$ lines in $L * L$, so $L^{\prime}$ contains at least $|L| n^{-2 \alpha}$ lines.

Uniformly at random choose a subset $R \subseteq L^{\prime}$ of $\left\lceil\left|L^{\prime}\right| n^{-c \alpha}\right\rceil$ lines, where $c>0$ is a parameter to be chosen later. The probability that a star family $S$ in $L^{\prime}$ contains at least $k$ lines of $R$ is

$$
\frac{\left(\begin{array}{c}
|S| \\
k
\end{array}\right) \cdot\left(\begin{array}{c}
\left|L^{\prime}\right|-k \\
|R|-k
\end{array}\right)}{\left(\begin{array}{c}
\left|L^{\prime}\right| \\
|R|
\end{array}\right)} \leq \frac{n^{k \alpha}}{k !} \cdot \frac{\left(\begin{array}{c}
\left|L^{\prime}\right| \\
|R|-k
\end{array}\right)}{\left(\begin{array}{c}
\left|L^{\prime}\right| \\
|R|
\end{array}\right)} .
$$


Applying Lemma 16 with $x=\left|L^{\prime}\right|$ and $x^{1-\gamma}=|R|=x^{1-c \alpha \log _{x}(n)}$, for large values of $n$ we have

$$
\frac{\left(\begin{array}{c}
\left|L^{\prime}\right| \\
|R|-k
\end{array}\right)}{\left(\begin{array}{c}
\left|L^{\prime}\right| \\
|R|
\end{array}\right)}=(1+o(1)) \cdot\left|L^{\prime}\right|^{-k c \alpha \log _{\left|L^{\prime}\right|}(n)} \leq 2 n^{-k c \alpha} .
$$

Since there are at most $n^{2 \varepsilon}$ star families, the expected number of star families with at least $k$ lines of $R$ is bounded by

$$
\frac{2}{k !} n^{2 \varepsilon+k(1-c) \alpha}
$$

Taking $k=\left\lceil\frac{\varepsilon}{\alpha}\right\rceil$ and $c=3$ makes this expected value less than 1 , meaning there is some choice of $R$ such that no star family has more than $\lceil\varepsilon / \alpha\rceil$ lines in $R$.

Thus, $R$ is a near-general position subset of $L^{\prime}$ (and therefore of $L$ ) with size at least $\left|L^{\prime}\right| n^{-3 \alpha} \geq|L| n^{-5 \alpha}$.

We remark that the proof still holds if $L * L$ above is replaced by $L^{\prime \prime} \subseteq L * L$ so long as $\left|L^{\prime \prime}\right| \geq|L| n^{-c_{0} \alpha}$ for some $c_{0}>0$. We will use this modified version in the proof that Theorem 2 implies Theorem 1 .

\section{Proof of Theorem 2}

Using Corollary 15, we are now ready to prove Theorem 22. A major tool used will be the commutator graph, which we draw from [4].

Let $A \subseteq \mathbb{R}$, let $\delta>0$, and let $L$ be a set of $n^{1-\delta}$-rich lines in $\mathbb{R}^{2}$. The commutator graph on $L$ is the graph $G=(V, E)$, where

$$
V(G)=L * L \cup L^{-1} * L^{-1}
$$

(with the minor change that we require minimum richness only $n^{1-5 \delta}$ for each line in $L * L$ and $\left.L^{-1} * L^{-1}\right)$ and

$$
E(G)=\left\{\left\{f * g, g^{-1} * f^{-1}\right\}: f, g \in L, f * g \in L * L, g^{-1} * f^{-1} \in L^{-1} * L^{-1}\right\} .
$$

We draw attention to the fact that the lines $f * g$ and $g^{-1} * f^{-1}$ have the same slope. Hence, any edge of the commutator graph is between two parallel lines.

Proof of Theorem 2. Let $\varepsilon>0$, let $0<\delta \ll \varepsilon$, and let $A \subset \mathbb{R}$ with $n=|A|>0$. Suppose for a contradiction that $L$ is a set of at least $n^{1-\varepsilon}$ lines, all $n^{1-\delta}$-rich in $A \times A$, and that $L$ is in near-general position with star families bounded in size by a constant $C>0$ independent of $n$. Consider the commutator graph on $L$.

If $|V(G)| \geq n^{1+4 \delta}$, then we contradict Theorem 3, so let us assume that $|V(G)|<n^{1+4 \delta}$. We claim that $|E(G)| \geq n^{2-6 \delta}$. If this is true, then there is a vertex with degree at least $|E(G)| /|V(G)|$, so there is a connected component (corresponding to a set of parallel lines) of size $n^{1-10 \delta}$, in contradiction with Theorem 11(i). 
Let $S(f)=X(f) \times Y(f)$ for each $f \in L$. By applying Lemma 8 to the collection of sets $S(f)$, where each set $S(f)$ has size at least $n^{2-2 \delta}$, we must have at least $n^{2-4 \delta} / 2 \geq n^{2-5 \delta}$ pairs $S(f), S(g)$ with $|S(f) \cap S(g)| \geq n^{2-4 \delta} / 2 \geq n^{2-5 \delta}$. Note that for any sets $A_{1}, A_{2}, A_{3}, A_{4}$, $\left(A_{1} \times A_{3}\right) \cap\left(A_{2} \times A_{4}\right)=\left(A_{1} \cap A_{2}\right) \times\left(A_{3} \cap A_{4}\right)$. Thus, since $|S(f) \cap S(g)| \geq n^{2-5 \delta}$, we have $|X(f) \cap X(g)| \geq n^{1-5 \delta}$ and $|Y(f) \cap Y(g)| \geq n^{1-5 \delta}$. Thus, we have at least $n^{2-5 \delta}$ pairs $f, g \in L$ such that $f * g$ and $g^{-1} * f^{-1}$ are each $n^{1-5 \delta}$-rich.

Let $f_{i}, g_{i}$ denote the lines such that $P_{i}:=\left\{f_{i} * g_{i}, g_{i}^{-1} * f_{i}^{-1}\right\}$ is a pair of $n^{1-5 \delta}$-rich lines. Given an index $i, f_{i}$ and $g_{i}$ intersect at a unique point $(x, y)$; it then follows that $y$ is the unique fixed point of $f_{i} * g_{i}$ and $x$ is the unique fixed point of $g_{i}^{-1} * f_{i}^{-1}$. Suppose there were $2 C+2$ indices $i_{1}, \ldots, i_{2 C+2}$ such that $P_{i_{j}}=P_{i_{k}}$ for all $1 \leq j, k \leq 2 C+2$. Then there would exist $C+1$ indices $i_{j_{1}}, \ldots, i_{j_{C+1}}$ such that

$$
f_{i_{j_{1}}} * g_{i_{j_{1}}}=\cdots=f_{i_{C+1}} * g_{i_{C+1}} \quad \text { and } \quad g_{i_{j_{1}}}^{-1} * f_{i_{j_{1}}}^{-1}=\cdots=g_{i_{C+1}}^{-1} * f_{i_{C+1}}^{-1} .
$$

Since for each $1 \leq k \leq C+1$ there is a unique $(x, y)$ such that $f_{i_{j_{k}}} * g_{i_{j_{k}}}(y)=y$ and $g_{i_{j_{k}}}^{-1} * f_{i_{j_{k}}}^{-1}(x)=x$, it follows that $f_{i_{j_{k}}}$ and $g_{i_{j_{k}}}$ all intersect the point $(x, y)$. Since the $f_{i_{j_{k}}} * g_{i_{j_{k}}}$ must all have the same slope and $L$ has no parallel lines, we cannot have that $f_{i_{j_{k}}}=f_{i_{j_{k}^{\prime}}}$ for $k \neq k^{\prime}$ or else $g_{i_{j_{k}}}=g_{i_{j_{k}^{\prime}}}$ as well, contradicting distinctness of the pairs. Similarly we must have $g_{i_{j_{k}}} \neq g_{i_{j_{k}^{\prime}}}$ for $k \neq k^{\prime}$. The collection

$$
\left\{f_{i_{j_{k}}}: 1 \leq k \leq C+1\right\} \cup\left\{g_{i_{j_{k}}}: 1 \leq k \leq C+1\right\}
$$

must therefore contain at least $C+1$ distinct lines (a single line may appear as an $f_{i_{j}}$ at most once and as a $g_{i_{j}}$ at most once). But then we have a set of more than $C$ concurrent lines at $(x, y)$, contradicting the hypothesis that $L$ is in almost-general position.

Thus, for each edge $e$, there are at most $2 C+2$ pairs $\left\{f_{i} \circ g_{i}^{-1}, g_{i}^{-1} \circ f_{i}\right\}$ equal to $e$, so the total number of edges in $G$ is at least $n^{2-5 \delta} /(2 C+2) \gg n^{2-6 \delta}$, yielding a contradiction with Theorem 11(i).

We remark that taking $\delta<\varepsilon / 12$ is sufficient for the proof to go through.

\section{Theorem 2 Implies TheOrem 1}

For $\ell \in L * L$, recall that $\mathcal{P}(\ell)$ is the set of all pairs $(f, g) \in L \times L$ such that $f * g=\ell$.

Lemma 17. For all $0<\varepsilon<1$, there exists $0<\alpha_{0}<\varepsilon$ such that, for all $0<\alpha<\alpha_{0}$, there exists $0<\delta_{0}<\alpha$ such that for all $0<\delta<\delta_{0}$ and for sufficiently large $n$, the following holds:

Let $A \subseteq \mathbb{R}$ have size $n$, and let $L$ be a set of at least $n^{\varepsilon}$ near-general position lines, all of which are $n^{1-\delta}$-rich in $A \times A$. Then there exists a set $L^{\prime} \subseteq L * L$ such that $L^{\prime}$ is a set of 
lines in near-general position, $\left|L^{\prime}\right|>|L| n^{-5 \alpha-4 \delta}$, and for all $\ell \in L^{\prime}$,

$$
|\mathcal{P}(\ell)| \geq \frac{|L|^{2}}{2|L * L| n^{3 \delta}} .
$$

Proof. Let

$$
S:=\left\{(f, g) \in L \times L: f * g \text { is } n^{1-5 \delta} \text {-rich }\right\} .
$$

By Lemma $9,|S| \geq|L|^{2} n^{-3 \delta}$. Let

$$
T:=\left\{(f, g) \in S:|\mathcal{P}(f * g)| \leq \frac{|L|^{2}}{2|L * L| n^{3 \delta}}\right\} .
$$

If $|T|>|S| / 2$, then we obtain an absurdity:

$$
\begin{aligned}
|L * L|=\sum_{(f, g) \in S} \frac{1}{|\mathcal{P}(f * g)|}=\sum_{(f, g) \in S \backslash T} \frac{1}{|\mathcal{P}(f * g)|} & +\sum_{(f, g) \in T} \frac{1}{|\mathcal{P}(f * g)|} \geq \\
& \frac{1}{|L|}|S \backslash T|+\frac{2|L * L| n^{3 \delta}}{|L|^{2}}|T|>|L * L| .
\end{aligned}
$$

Thus, $|S \backslash T| \geq|L|^{2} n^{-3 \delta} / 2>|L|^{2} n^{-4 \delta}$. Letting $L^{\prime}=\{f * g:(f, g) \in S \backslash T\}$, we then have $\left|L^{\prime}\right| \geq|L| n^{-4 \delta}$. Apply Corollary 15 to deduce that $L^{\prime}$ contains a subset of $|L| n^{-5 \alpha-4 \delta}$ lines in near-general position.

Proposition 18. Theorem 2 implies Theorem 1 .

Proof. Let $L$ be a set of $n^{\varepsilon}$ lines in general position, all of which are $n^{1-\delta}$-rich for some $\delta>0$ to be chosen later. Fix $\alpha<\varepsilon$, and suppose $\left|L^{*(k+1)}\right| \geq\left|L^{* k}\right| n^{5 \alpha}$ for all $k$ up to $m=\lfloor 2 / \alpha\rfloor$. (By Corollary 15, we may further assume that $L^{* j}$ is in near-general position for all $j \leq k$ at the cost of a factor of $n^{4 \alpha}$ each iteration.) Redefining $\delta$ if necessary, we can take $1-4 \cdot 5^{m} \delta>0$. For sufficiently large $n$, we then have

$$
\left|L^{*(m+1)}\right| \geq n^{\varepsilon+m \alpha} \geq n^{2} .
$$

But this violates Theorem 3, so such an $m$ cannot exist. Therefore there exists $k<2 / \alpha$ such that

$$
\left|L^{*(k+1)}\right|<\left|L^{* k}\right| n^{5 \alpha} \text {. }
$$

In this case, let $L^{\prime}=L^{* k}$ for the smallest such $k$ (such that the above inequality would now read $\left.\left|L^{\prime} * L^{\prime}\right|<\left|L^{\prime}\right| n^{5 \alpha}\right)$, let $\alpha^{\prime}<5 \alpha$ such that $\alpha^{\prime} \ll \varepsilon$, let $N=\left|L^{\prime}\right|$, and choose $\delta^{\prime} \leq 5^{k} \delta$ such that $\delta^{\prime} \ll \alpha^{\prime}$.

By applying Lemma 17, we can restrict our attention to a subset $L^{\prime \prime} \subseteq L^{\prime} * L^{\prime}$ of size at least $N n^{-5 \alpha^{\prime}-4 \delta^{\prime}}$ such that all lines in $L^{\prime \prime}$ are in near-general position and, for all $\ell \in L^{\prime \prime}$,

$$
|\mathcal{P}(\ell)| \geq \frac{N^{2}}{2\left|L^{\prime} * L^{\prime}\right| n^{3 \delta^{\prime}}} \geq \frac{N}{2 n^{5 \alpha+3 \delta^{\prime}}}>\frac{N}{2 n^{\alpha^{\prime}+3 \delta}} .
$$


If $\ell$ is a line in $L^{\prime \prime}$, then $\ell=f * g$ for some $f, g \in L^{\prime}$. We will then have at least

$$
\frac{1}{C}\left|L^{\prime \prime}\right|\left(N n^{-\alpha^{\prime}-4 \delta^{\prime}}\right)^{2} \geq \frac{1}{C} N^{3} n^{-5 \alpha^{\prime}-8 \delta^{\prime}} \gg N^{3} n^{-6 \alpha^{\prime}}
$$

solutions $\left(f, g, f^{\prime}, g^{\prime}\right) \in L \times L \times L \times L$ to the equation

$$
f^{\prime} * f(0)=g^{\prime} * g(0)
$$

(The factor of $1 / C$ comes from the fact that $L^{\prime \prime}$ is a set of lines in near-general position, so at most $C$ lines will share a $y$-intercept.)

Now, fixing $f^{\prime}, g^{\prime}$ in (5) and letting $f, g$ vary, we can interpret (5) as the line $f^{\prime} * g^{\prime}$, where the $x$ and $y$ variables are the $y$-intercepts of $f$ and $g$. Letting $B$ be the set of $y$-intercepts among lines in $L^{\prime \prime}$, we may interpret the above count of solutions to (5) as stating that many of the lines $f^{\prime} * g^{\prime}$ are $N n^{-7 \alpha^{\prime}}$-rich in the new grid $B \times B$. Indeed, let

$$
S:=\left\{\left(f^{\prime}, g^{\prime}\right) \in L^{\prime} \times L^{\prime}: f^{\prime} * g^{\prime} \text { is } N n^{-7 \alpha^{\prime}} \text {-rich in } B \times B\right\},
$$

and let $p\left(f^{\prime}, g^{\prime}\right)$ denote the number of points that $f^{\prime} * g^{\prime}$ intersects in $B \times B$. Then, for a contradiction, assume $|S|<N^{2} n^{-8 \alpha^{\prime}}$. This implies the absurdity:

$$
\begin{aligned}
N^{3} n^{-6 \alpha^{\prime}}= & \sum_{\left(f^{\prime}, g^{\prime}\right) \in S} p\left(f^{\prime}, g^{\prime}\right)+\sum_{\left(f^{\prime}, g^{\prime}\right) \in S^{c}} p\left(f^{\prime}, g^{\prime}\right)<|S||B|+\left|S^{c}\right|\left(N n^{-7 \alpha^{\prime}}\right)< \\
& |S| N n^{\alpha^{\prime}}+\left(N^{2}-|S|\right) N n^{-7 \alpha^{\prime}}<|S| N n^{\alpha^{\prime}}+N^{3} n^{-7 \alpha^{\prime}}<2 N^{3} n^{-7 \alpha^{\prime}} \ll N^{3} n^{-6 \alpha^{\prime}} .
\end{aligned}
$$

(Note that this requires $N \gg n^{4 \alpha^{\prime}}$, which is satisfied provided $\alpha^{\prime} \ll \varepsilon$ because $N \gg n^{\varepsilon}$.) Thus, $|S| \geq N^{2} n^{-8 \alpha^{\prime}}$, and that implies that we have at least $N n^{-8 \alpha^{\prime}}$ lines that are all $N n^{-7 \alpha^{\prime}}$ rich in $B \times B$. Moreover, since $L^{\prime}$ is in near-general position, we may extract a set of lines from $S * S$ that are in near-general position, and this set has size at least $N n^{-11 \alpha^{\prime}}$. However, this is in contradiction with Theorem 2, since $S * S$ is a set of $N^{1-\gamma}$-rich lines in near-general position for some $\gamma>0$, and $|S * S| \geq N^{1-12 \gamma}$.

\section{Connections to Freiman's Theorem}

Theorem 1 implies a statistical version of a Freiman-type theorem on linear functions with small image sets. We primarily base the following exposition on selected sections of Elekes' survey paper, [4]. We direct the reader to the references and proofs therein.

A proper generalized arithmetic progression, or GAP, $G \subseteq \mathbb{R}$ is a set of the form

$$
G=\left\{a_{0}+r_{1} a_{1}+\cdots+r_{d} a_{d}: 0 \leq r_{1} \leq n_{1}, \ldots, 0 \leq r_{d} \leq n_{d}\right\},
$$

where $a_{0}, \ldots, a_{d} \in \mathbb{R}, n_{1}, \ldots, n_{d} \in \mathbb{Z}^{+}$, and $|G|=n_{1} \cdots n_{d}$. An analogous definition may be made for a proper generalized geometric progression, or GGP, $G \subseteq \mathbb{R} \backslash\{0\}$, where

$$
G=\left\{a_{0} \cdot r_{1} a_{1} \cdots r_{d} a_{d}: 0 \leq r_{1} \leq n_{1}, \ldots, 0 \leq r_{d} \leq n_{d}\right\},
$$


where $a_{0}, \ldots, a_{d} \in \mathbb{R} \backslash\{0\}, n_{1}, \ldots, n_{d} \in \mathbb{Z}^{+}$, and $|G|=n_{1} \cdots n_{d}$. In both these cases, the dimension of $G$ is defined to be the minimal value of $d$ here, given $G$.

Recall Freiman's theorem on GAPs in the real numbers:

Theorem 19 (Freiman). Let $X, Y \subset \mathbb{R}$. If $|X|,|Y| \geq n$ and $|X+Y| \leq$ cn, then $X \cup Y$ is contained in a GAP of dimension at most $d=d(c)$ and size at most $C n, C=C(c)$ (that is, $d$ and $C$ do not depend on $n$ ).

This theorem holds for GGPs in the reals as well.

If $X \subset \mathbb{R}$, let $E$ be a set of unordered pairs of elements in $X$. In this way, $E$ can be considered the edge set of a graph $G$ with vertex set $X$. We define

$$
X+{ }_{E} X:=\left\{x^{\prime}+x^{\prime \prime}:\left\{x^{\prime}, x^{\prime \prime}\right\} \in E\right\} .
$$

Theorem 20 (Balog, Szemerédi). If $|E| \geq a|X|^{2}$ and $\left|X+_{E} X\right| \leq c|X|$, then there exists $\alpha=\alpha(a, c)$ such that some $\alpha|X|$ elements of $X$ are contained in a GAP of dimension $d=d(a, c)$ and size $C|X|, C=C(a, c)$.

Elekes [4] describes this version of the Balog-Szemerédi theorem as a "statistical" version of Freiman's theorem. He further observes that a "uniform statistical" hypothesis (that is, requiring a minimum degree proportional to $|X|$ in the graph $G$ ) guarantees that $X$ can be covered by a constant number of GAPs.

Theorem 21 (Elekes, Ruzsa). Let $X \subset \mathbb{R}$ be finite, $\alpha>0$ fixed, and $G$ a graph on $X$ with minimum degree $\alpha|X|$. Suppose that $\left|X+_{E} X\right| \leq c|X|$. Then, for all $\varepsilon>0$, $X$ can be partitioned into disjoint sets $X_{1}, \ldots, X_{k}$ with the following properties:

(1) Each $X_{i}$ is contained in a GAP $G_{i}$ of dimensiond and size $C|X|$ (the GAPs $G_{i}$ need not be disjoint);

(2) Each $X_{i}$ spans at least $\gamma|X|^{2}$ edges of $E$ (which implies that $k \leq 1 / \gamma$ ); and

(3) There are at most $\varepsilon|X|^{2}$ "leftover" edges outside the $X_{i}$ :

$$
\sum_{i<j} \sum_{x^{\prime} \in X_{i}} \sum_{x^{\prime \prime} \in X_{j}} \mathbf{1}_{E}\left(\left\{x^{\prime}, x^{\prime \prime}\right\}\right) \leq \varepsilon|X|^{2},
$$

where $d=d(a, c, \varepsilon), C=C(\alpha, c, \varepsilon)$, and $\gamma=\gamma(\alpha, c)$.

It turns out that Freiman's theorem extends to more general objects than sets of real numbers: sets of linear functions. Elekes observes that sets of lines with small composition sets satisfy a particular "two extremities" structure.

Let $\mathcal{L}$ be the set of (affine) nonconstant linear functions $\mathbb{R} \rightarrow \mathbb{R}$.

Theorem 22 (Elekes [4]). For all $c, C>0$, there exists $c^{\prime}=c^{\prime}(c, C)>0$ with the following property. 
Let $\Phi, \Psi \subset \mathcal{L}$ be sets of $n$ lines each and $E \subset \Phi \times \Psi$ have size at least $c n^{2}$. Define

$$
\Phi \circ_{E} \Psi:=\{\phi \circ \psi:(\phi, \psi) \in E\} .
$$

If $\left|\Phi \circ_{E} \Psi\right| \leq C n$, then there exist subsets $\Phi^{\prime} \subseteq \Phi$ and $\Psi^{\prime} \subseteq \Psi$ such that

$$
\left|\left(\Phi^{\prime} \times \Psi^{\prime}\right) \cap E\right| \geq c^{\prime} n^{2}
$$

and either

(i) both $\Phi^{\prime}$ and $\Psi^{\prime}$ consist of functions whose graphs are all parallel (but the directions may be different for $\Phi^{\prime}$ and $\left.\Psi^{\prime}\right)$; or

(ii) both $\Phi^{\prime}$ and $\Psi^{\prime}$ consist of functions whose graphs all pass through a common point (which may be different for $\Phi^{\prime}$ and $\Psi^{\prime}$ ).

Using this theorem along with Theorem 19, Elekes proves a result analogous to Theorem 19: in fact, a true generalization of Freiman's theorem.

Theorem 23 (Elekes [4]). For every $C>0$ there are $C^{\prime}=C^{\prime}(C)>0, C^{\prime \prime}=C^{\prime \prime}(C)>0$, and $d=d(C)$ with the following property.

If $\Phi, \Psi \subset \mathcal{L}$ are sets of $n$ lines each and $|\Phi \circ \Psi| \leq C n$, then $\Phi^{-1} \cup \Psi$ is contained in a union of $C^{\prime}$ parallel families or a union of $C^{\prime}$ star families, and each of those families has size at most $C^{\prime \prime} n$.

Elekes further notes that a uniform statistical version of this Freiman-type theorem for linear functions can be deduced from Theorem 21 .

Theorem 24 (Elekes [4]). Let $\alpha>0$ be fixed, $\Phi, \Psi$ as in Theorem 23, and $G(\Phi, \Psi, E) a$ bipartite graph with minimum degree at least $\alpha$. If $\left|\Phi \circ_{E} \Psi\right| \leq C n$, then $\Phi^{-1} \cup \Psi$ is the union of a constant number of parallel and star families.

The previous theorems have focused on sets of lines $\Phi, \Psi$ whose composition set $\Phi \circ \Psi$ is small. Another direction we can explore is to study sets of lines $\Phi$ and points $H \subset \mathbb{R}$ whose image set $\Phi(H)$ is small. In particular, Theorem 22 is equivalent to the following:

Theorem 25 (Elekes [4]). For all $c, C>0$, there exists $c^{\prime}=c^{\prime}(c, C)>0$ with the following property.

Let $H \subset \mathbb{R}$ and $\Phi \subset \mathcal{L}$ have size at least $n$, let $E \subset \Phi \times H$ have size at least $\mathrm{cn}^{2}$, and define

$$
\Phi_{E}(H):=\{\phi(h):(\phi, h) \in E\} .
$$

If $\left|\Phi_{E}(H)\right| \leq C n$, then there exists a parallel or star family $\Phi^{\prime} \subseteq \Phi$ such that

$$
\left|E \cap\left(\Phi^{\prime} \times H\right)\right| \geq c^{\prime} n^{2}
$$


A result about small image sets analogous to Theorem 23 holds:

Theorem 26 (Elekes [4]). For every $C>0$ there are $C^{\prime}=C^{\prime}(C)>0$ and $d=d(C)>0$ with the following property.

Let $\Phi \subset \mathcal{L}$ be a set of $n$ lines and $H \subset \mathbb{R}$ be a set of $n$ points. If $|\Phi(H)| \leq C n$, then $\Phi$ is contained in union of at most $C^{\prime}$ parallel families or a union of at most $C^{\prime}$ star families, and each of these families has size at most $C^{\prime \prime} n$.

Elekes conjectured that a uniform statistical version of this theorem similar to Theorem 24 holds. We study one such statement in which there is a minimum degree requirement on only one side of the bipartite graph.

Conjecture 27 (Elekes [4]). Let $\alpha>0$ be fixed, $\Phi, H$ as in Theorem 25, and $G(\Phi, H, E) a$ bipartite graph with degree at least an for each $\phi \in \Phi$. If $\left|\Phi_{E}(H)\right| \leq C n$, then $\Phi$ is the union of a constant number of parallel and star families.

In terms of cartesian products, this conjecture is equivalent to the following:

Conjecture 28 (Elekes [4]). If each of cn lines is cn-rich in an $n \times n$ cartesian product, then the set of lines is the union of $C=C(c)$ parallel and star families.

The following proof is not given explicitly in [4] but can be inferred from similar arguments presented in that paper.

Proof of Equivalence. Suppose Conjecture 27 holds. Fix $0<c<1$, and let $\Phi$ be a set of cn lines, each $c n$-rich in a $n \times n$ Cartesian product $A \times B$. Construct the bipartite graph $G(\Phi, A, E)$, where an edge connects $\phi \in \Phi$ and $a \in A$ whenever $\phi(a) \in B$. Then the degree of each $\phi \in \Phi$ is at least $c n$, so $\Phi$ is the union of a constant number of parallel and star families.

For the other implication, suppose we have a set of $N$ lines $\Phi$, a set of $n$ points $H$, and an edge set $E \subseteq \Phi \times H$ such that $\left|\Phi_{E}(H)\right| \leq C n$ for $C>0$. Observe that each line from $\Phi$ occurs in at least $\alpha n$ pairs of $E$, so we have $n \geq \alpha n$ lines which are each $\alpha n$-rich in the cartesian product $\left(H \cup \Phi_{E}(H)\right) \times\left(H \cup \Phi_{E}(H)\right)$, which has size at most $(C+1)^{2} N^{2}$. So the lines are the union of a constant number of parallel and star families.

Conjecture 28, and therefore Conjecture 27, would be implied by following conjecture of József Solymosi:

Conjecture 29 (Solymosi [4]). Among the lines which are cn-rich in an $n \times n$ cartesian product, at most $C=C(c)$ can be in general position.

Proof that Conjecture 29 implies Conjecture 28. Given a set $L$ of $c n$ lines which are $c n$-rich in an $n \times n$ grid, let $L^{\prime}$ be a maximum collection of these lines in general position. By 
Conjecture 29, $\left|L^{\prime}\right|=C$ for some constant $C$. Define $L(\lambda)$ to be the set of lines in $L$ with slope $\lambda$ and define $L(p)$ to be the set of lines in $L$ passing through a given point $p \in \mathbb{R}^{2}$. Then the union of $L(\lambda)$ over all $\lambda$ which are slopes of lines in $L^{\prime}$ and $L(p)$ over all points $p$ which are intersections of pairs of lines in $L^{\prime}$ must be $L$. So $L$ is the union of $C+C^{2}$ parallel and star families.

The main result of the present paper, Theorem 1, yields an analogous result to Conjecture 28.

Corollary 30. For every $\varepsilon>0$, there exists $0<\delta_{0}<\varepsilon$ such that for all $0<\delta<\delta_{0}$, the following holds for sufficiently large $n$ :

A set of lines that are each $n^{1-\delta}$-rich in an $n \times n$ cartesian product must be the union of $n^{\varepsilon}$ parallel and star families.

Proof. Given $\varepsilon>0$, choose $\delta>0$ to satisfy Theorem 11. Then given a set $L$ of lines which are $n^{1-\delta}$-rich in an $n \times n$ grid, let $L^{\prime}$ be a maximum collection of these lines in general position. So $\left|L^{\prime}\right| \leq n^{\varepsilon}$ for some constant $\varepsilon>0$. Define $L(\lambda)$ to be the set of lines in $L$ with slope $\lambda$ and define $L(p)$ to be the set of lines in $L$ passing through a given point $p \in \mathbb{R}^{2}$. Then the union of $L(\lambda)$ over all $\lambda$ which are slopes of lines in $L^{\prime}$ and $L(p)$ over all points $p$ which are intersections of pairs of lines in $L^{\prime}$ must be $L$. So $L$ is the union of $n^{\varepsilon}+n^{2 \varepsilon}$ parallel and star families.

Therefore, we also have the following uniform statistical Freiman-type theorem similar to Conjecture 27:

Corollary 31. For every $\varepsilon>0$, there exists $0<\delta_{0}<\varepsilon$ such that for all $0<\delta<\delta_{0}$, the following holds for sufficiently large $n$ :

Let $\Phi, H$ be as in Theorem 25 and $G(\Phi, H, E)$ be a bipartite graph with degree at least $n^{1-\delta}$ for each $\phi \in \Phi$. If $\left|\Phi_{E}(H)\right| \leq n^{1+\delta}$, then $\Phi$ is the union of $n^{\varepsilon}$ parallel and star families.

Proof. Let $A=H \cup \Phi_{E}(H)$. Each line in $\Phi$ is incident with at least $n^{1-\delta}$ edges of $E$, so each of the $n$ lines of $\Phi$ is $n^{1-\delta}$-rich in the $n^{1+\delta} \times n^{1+\delta}$ cartesian product $A \times A$. In other words, each line of $\Phi$ is $|A|^{1-\delta^{\prime}}$-rich in $A \times A$, where $\delta^{\prime}=1-\frac{1-\delta}{1+\delta}$. Since $\delta^{\prime} \rightarrow 0$ as $\delta \rightarrow 0$, by choosing $\delta$ small enough, we can ensure through Corollary 30 that $\Phi$ is the union of $n^{\varepsilon}$ parallel and star families.

\section{REFERENCES}

[1] E. Borenstein and E. Croot, "On rich lines in grids," Discrete E Comp. Geom. 43 (2010) 824-840.

[2] E. Borenstein and E. Croot, "On a certain generalization of the Balog-Szemerédi-Gowers theorem," SIAM J. Discrete Math. 25 (2011), 685-694. 
[3] E. Croot and D. Hart, " $h$-fold sums from a set with few products," SIAM J. Discrete Math. 24 (2010) $505-519$.

[4] Gy. Elekes, "Sums versus products in number theory, algebra and Erdös geometry," Paul Erdös and his mathematics, II (Budapest, 1999), Bolyai Soc. Math. Stud. 11 (ed. G. Halász, Jànos Bolyai Math. Soc., Budapest, 2002) 241-290.

[5] E. Szemerédi and W. Trotter, "Extremal problems in discrete geometry," Combinatorica 3 (1983) 381392.

[6] T. Tao and V. Vu, Additive Combinatorics, Cambridge University Press (2010). 